\title{
ORDINAL RANKINGS ON MEASURES ANNIHILATING THIN SETS
}

\author{
ALEXANDER S. KECHRIS AND RUSSELL LYONS
}

\begin{abstract}
We assign a countable ordinal number to each probability measure which annihilates all $H$-sets. The descriptive-set theoretic structure of this assignment allows us to show that this class of measures is coanalytic non-Borel. In addition, it allows us to quantify the failure of Rajchman's conjecture. Similar results are obtained for measures annihilating Dirichlet sets.
\end{abstract}

A closet subset $E$ of the unit circle $\mathbf{T}=\mathbf{R} / \mathbf{Z}$ is called an $H$-set if there exists a sequence $\left\{n_{k}\right\}$ of positive integers tending to $\infty$ and an interval (i.e., a nonempty open arc) $I \subseteq \mathrm{T}$ such that for all $k$ and all $x \in E, n_{k} x \notin I$. These sets play a fundamental role as examples of sets of uniqueness for trigonometric series [KL; Z, Chapters IX, XII]. A (Borel) probability measure $\mu$ on $\mathbf{T}$ is called a Rajchman measure if $\hat{\mu}(n) \rightarrow 0$ as $|n| \rightarrow \infty$, where $\hat{\mu}(n)=\int_{T} e(-n x) d \mu(x), e(x)=e^{2 \pi i x}$. We denote by $R$ the class of such measures. These measures have also been very important to the study of sets of uniqueness. In particular, every Rajchman measure annihilates every set of uniqueness, hence every $H$-set. After establishing these relationships [R1, R2], Rajchman conjectured that, in fact, the only measures which annihilate all $H$-sets are those in $R$. This, however, is false $[\mathbf{L 1}, \mathbf{L 2}, \mathbf{L 3}$, L5]. Here, we shall quantify how distant Rajchman's conjecture is from the truth.

Given a class $\mathscr{C}$ of closed subsets of $\mathbf{T}$, denote by $\mathscr{C}^{\perp}$ the class of probability measures on $\mathbf{T}$ which annihilate all sets in $\mathscr{C}: \mu \in \mathscr{C}^{\perp} \Leftrightarrow \forall E \in \mathscr{C}(\mu(E)=0)$. Thus $R \varsubsetneqq H^{\perp}$, where $H$ denotes the class of $H$-sets. Denote by PROB(T) the compact, metrizable space of (Borel) probability measures on $\mathbf{T}$ with the weak ${ }^{*}$ topology. It is easy to check that $R$ is a Borel, in fact $\Pi_{3}^{0}$ (i.e., $F_{\sigma \delta}$ ), subset of this space. We establish in $\S 3$ that $H^{\perp}$ is a $\Pi_{1}^{1}$ (i.e., coanalytic) but not Borel subset of $\operatorname{PROB}(\mathbf{T})$. This is the first example of a natural class of measures of such complexity known to the authors and it highlights the distinction between $R$ and $H^{\perp}$.

Our method of proof actually provides quite a bit of further information on the relationship between $R$ and $H^{\perp}$. In $\S 1$, we assign to each $\mu \in H^{\perp}$ a countable ordinal number $h(\mu)$ which measures in some sense the complexity of the verification that $\mu$ annihilates all $H$-sets. We show that $h$ has certain definability properties, namely, it is a $\Pi_{1}^{1}$-rank (see [KL]). In $\S 3$, using the techniques developed in [L3 and L4], we prove that the rank $h$ is unbounded in $\omega_{1}$, the first uncountable ordinal;

Received by the editors September 10, 1987.

1980 Mathematics Subject Classification (1985 Revision). Primary 43A46, 03E15; Secondary 43A05, 42A63, 54H05.

Key words and phrases. Rajchman measures, thin sets, sets of uniqueness, rank.

Research of the first author partially supported by NSF grant DMS8718847.

The second author was partially supported by an NSF Postdoctoral Fellowship. 
that is, $\forall \alpha<\omega_{1} \exists \mu \in H^{\perp}(h(\mu) \geq \alpha)$. It follows from the Boundedness Theorem for $\Pi_{1}^{1}$-ranks (see [KL, V.1]) that $H^{\perp}$ is not Borel.

Denote by $H_{\alpha}^{\perp}$ the class of all $\mu \in H^{\perp}$ with $h(\mu) \leq \alpha$. Thus $H_{1}^{\perp} \subseteq H_{2}^{\perp} \subseteq$ $\cdots \subseteq H_{\alpha}^{\perp} \subseteq \cdots \subseteq H_{\beta}^{\perp} \ldots\left(\alpha<\beta<\omega_{1}\right), H^{\perp}=\bigcup_{\alpha<\omega_{1}} H_{\alpha}^{\perp}$ and $H_{\alpha}^{\perp} \subsetneq H^{\perp}$ for each $\alpha<\omega_{1}$, so that $\left\{H_{\alpha}^{\perp}\right\}$ is a stratification of $H^{\perp}$ in a hierarchy of increasing complexity. The "simplest" measures in $H^{\perp}$ are those in $H_{1}^{\perp}$. In $\S 2$, we prove that these include the Rajchman measures: $R \subseteq H_{1}^{\perp}$. This bound gives quantitative meaning to the statement that $R$ is only a small part of $H^{\perp}$. We also show that another canonical class of measures in $H^{\perp}$, the so-called quasisymmetric measures, belong to $H_{\omega}^{\perp}$. We do not know if they belong to $H_{1}^{\perp}$. An interesting consequence of these upper bounds is that the techniques in [L3] for proving measures to be in $H^{\perp}$ are demonstrated to be more powerful in a quantitative sense than the traditional techniques used (and amplified) in $\S 2$.

We conclude this paper by establishing, in $\S 4$, analogous results for the class $D^{\perp}$ of probability measures annihilating all Dirichlet sets. (Recall that a closed set $E \subseteq \mathbf{T}$ is a Dirichlet set $[\mathbf{L P}]$ if there is a sequence of positive integers $\left\{n_{k}\right\}$ tending to $\infty$ such that $\sup _{x \in E}\left\|n_{k} x\right\| \rightarrow 0$, where $\|x\|=\operatorname{dist}(x, \mathbf{Z})$.) $D^{\perp}$ has been studied before under the name $\mathscr{L}_{I}$ (see [HMP, pp. 212-215, 242-247]).

NOTE. After completion of this manuscript, we learned that B. Host, A. Louveau, and F. Parreau established several months earlier than us that $D^{\perp}$ is not Borel; they used the characterization of $D^{\perp}$ given on p. 243 of [HMP]. Their work is unpublished.

1. A $\Pi_{1}^{1}$-rank on $H^{\perp}$. Let $K(\mathbf{T})$ be the space of closed subsets of $\mathbf{T}$ with the standard Hausdorff metric. This is a compact space. As a subset of $K(\mathbf{T})$, it is verified in [KL, IV.2.7] that $H$ is $\Sigma_{3}^{0}$ (i.e., $G_{\delta \sigma}$ ).

We next compute the following upper bound for the complexity of $H^{\perp}$.

PROPOSITION 1.1. The set $H^{\perp}$ is $\boldsymbol{\Pi}_{1}^{1}$ (i.e., coanalytic) in the space $\operatorname{PROB}(\mathbf{T})$.

Proof. Since $H$ is Borel and

$$
\mu \in H^{\perp} \Leftrightarrow \forall E \in K(\mathbf{T}) \quad[E \in H \Rightarrow \mu(E)=0]
$$

for $\mu \in \operatorname{PROB}(\mathbf{T})$, it is enough to show that the relation

$$
\{(\mu, E): \mu \in \operatorname{PROB}(\mathbf{T}) \& E \in K(\mathbf{T}) \& \mu(E)=0\}
$$

is Borel (in fact, $G_{\delta}$ ) in the space $\operatorname{PROB}(\mathbf{T}) \times K(\mathbf{T})$. Now, if $\left\{V_{n}\right\}$ is an open basis for the topology of $\mathbf{T}$ which is closed under finite unions, then for $\mu \in \operatorname{PROB}(\mathbf{T})$ and $E \in K(\mathbf{T})$,

$$
\mu(E)=0 \Leftrightarrow \forall m \exists n\left(E \subseteq V_{n} \& \mu\left(\mathbf{T} \backslash \bar{V}_{n}\right)>\frac{m-1}{m}\right) .
$$

Since $\{E: E \subseteq V\}$ is open in $K(\mathbf{T})$ for each open $V \subseteq \mathbf{T}$, while $\{\mu: \mu(V)>a\}$ is open in $\operatorname{PROB}(\mathbf{T})$ for each $a \in \mathbf{R}$ and $V$ open in $\mathbf{T}$, the result follows.

Our main result is that $H^{\perp}$ is not Borel, thus determining exactly the complexity of $H^{\perp}$.

We will use below notation concerning finite sequences and trees as in [KL, IV.1]. We denote by $\mathbf{N}^{*}$ the set of positive integers. To define our rank $h: H^{\perp} \rightarrow \omega_{1}$, we 
associate first to each $\mu \in \operatorname{PROB}(\mathbf{T})$, each $I$ which is a rational interval in $\mathbf{T}$, and each $\varepsilon \in \mathbf{Q}^{+}=\{x \in \mathbf{Q}: x>0\}$, a tree $T_{\mu}^{I, \varepsilon}$ on Seq $\mathbf{N}^{*}$ as follows:

$$
\begin{array}{r}
T_{\mu}^{I, \varepsilon}=\left\{\left(n_{0}, n_{1}, \ldots, n_{k}\right): \forall i \leq k\left(n_{i} \in \mathbf{N}^{*}\right) \& \forall i<k\left(n_{i+1} / n_{i} \geq 2\right) \&\right. \\
\left.\mu\left(\left\{x \in \mathbf{T}: \forall i \leq k\left(n_{i} x \notin I\right)\right\}\right) \geq \varepsilon\right\} .
\end{array}
$$

Notice that if $I \supseteq I^{\prime}$ and $\varepsilon \geq \varepsilon^{\prime}$, then $T_{\mu}^{I, \varepsilon} \subseteq T_{\mu}^{I^{\prime}, \varepsilon^{\prime}}$.

REMARK. The condition " $n_{i+1} / n_{i} \geq 2$ ", instead of the expected one, " $n_{i+1}>$ $n_{i}$ ", has the effect of thinning down the trees for normalization purposes. For example, in Theorem 2.4 below, we obtain that $h(\mu)=1$ for Rajchman measures, rather than $h(\mu) \leq \omega$.

Proposition 1.2. Let $\mu \in \operatorname{PROB}(\mathbf{T})$. Then $\mu \in H^{\perp} \Leftrightarrow \forall I \forall \varepsilon\left(T_{\mu}^{I, \varepsilon}\right.$ is well founded).

ProOF. If for some $I, \varepsilon, T_{\mu}^{I, \varepsilon}$ has an infinite branch $n_{0}, n_{1}, n_{2}, \ldots$, then $\mu(E) \geq$ $\varepsilon>0$ for $E=\left\{x \in T: \forall i\left(n_{i} x \notin I\right)\right\}$. As $E \in H$, we have $\mu \notin H^{\perp}$. Conversely, if $\mu \notin H^{\perp}$, let $\varepsilon \in \mathbf{Q}^{+}, E \in H$ be such that $\mu(E) \geq \varepsilon$ and let $0<n_{0}<n_{1}<\cdots$ and $I$ be such that $n_{k} x \notin I$ for all $k \in \mathrm{N}, x \in E$. By going to a subsequence, we can assume that $n_{i+1} / n_{i} \geq 2$ for all $i$. Then $\left(n_{0}, n_{1}, \ldots, n_{k}\right) \in T_{\mu}^{I, \varepsilon}$ for all $k$, i.e., $T_{\mu}^{I, \varepsilon}$ is not well founded.

For each well-founded tree $T$, we define its height, $h t(T)$, as follows. First, for each $s \in T$, define its height in $T$, ht $(s, T)$, by

$$
\begin{aligned}
\operatorname{ht}(s, T) & =0 \text { if } s \text { is terminal in } T, \text { i.e., has no proper extension in } T, \\
\operatorname{ht}(s, T) & =\sup \left\{\operatorname{ht}\left(s^{\wedge}(y), T\right)+1: s^{\wedge}(y) \in T\right\} \\
& =\sup \{\operatorname{ht}(t, T)+1: t \in T, t \supsetneqq s\} \text { otherwise. }
\end{aligned}
$$

Then let $\operatorname{ht}(T)=\operatorname{ht}(\varnothing, T)$. (This definition is slightly different from that used in [KL, V.1], where one defines ht $(s, T)=1$ if $s$ is terminal in $T$.)

For each $\mu \in H^{\perp}$, define the rank function $h^{\prime}(\mu)=\sup \left\{\operatorname{ht}\left(T_{\mu}^{I, \varepsilon}\right)+1: I\right.$ a rational interval and $\left.\varepsilon \in \mathbf{Q}^{+}\right\}$. Clearly $h^{\prime}: H^{\perp} \rightarrow \omega_{1}$. We will show first that $h^{\prime}(\mu)$ is always a limit ordinal, thus of the form $\omega \cdot \alpha$ for some $\alpha<\omega_{1}$. We will then define $h(\mu)$ by $h^{\prime}(\mu)=\omega \cdot h(\mu)$.

LEMMA 1.3. For each $\mu \in H^{\perp}, h^{\prime}(\mu)$ is a limit ordinal.

Proof. First notice that $h^{\prime}(\mu) \geq \omega$ for all $\mu \in H^{\perp}$. Indeed, given any $0<$ $n_{0}<n_{1}<\cdots<n_{k}$ with $n_{i+1} / n_{i} \geq 2$, one can easily find $I$ and $\varepsilon$ such that $\left(n_{0}, \ldots, n_{k}\right) \in T_{\mu}^{I, \varepsilon}$. To complete the proof, it is enough to show that if $\mu \in H^{\perp}$, $h^{\prime}(\mu)>\omega \cdot \alpha(\alpha \geq 1)$, and $N \in \mathbf{N}^{*}$, then $h^{\prime}(\mu) \geq \omega \cdot \alpha+N$. Since $h^{\prime}(\mu)>\omega \cdot \alpha$, there are $I$ and $\varepsilon$ with $h t\left(T_{\mu}^{I, \varepsilon}\right) \geq \omega \cdot \alpha$. Fix next a sequence $0<n_{0}<\cdots<n_{N-1}$ with $n_{i+1} / n_{i} \geq 2$ and a rational interval $I^{\prime} \subseteq I$ such that $\mu(\{x: \forall i \leq N-1$ $\left.\left(n_{i} x \notin I^{\prime}\right)\right\} \geq 1-\varepsilon / 2$.

We claim that if $S=\left\{(n)^{\wedge} t \in T_{\mu}^{I, \varepsilon}: n \geq 2 n_{N-1}\right\} \cup\{\varnothing\}$, then $\mathrm{ht}(S) \geq \omega \cdot \alpha$. This is because for some $s \in T_{\mu}^{I, \varepsilon}$, we have ht $\left(s, T_{\mu}^{I, \varepsilon}\right)=\omega \cdot \alpha$ and thus $\sup \left\{\operatorname{ht}\left(s^{\wedge}(n), T_{\mu}^{I, \varepsilon}\right)+\right.$ $1\}=\omega \cdot \alpha$. Since the supremum is not attained and $s^{\wedge}(n)^{\wedge} t \in T_{\mu}^{I, \varepsilon} \Rightarrow(n)^{\wedge} t \in T_{\mu}^{I, \varepsilon}$, it follows that $\operatorname{ht}(S) \geq \omega \cdot \alpha$.

Now let

$$
T=\left\{\left(n_{0}, \ldots, n_{N-1}\right)^{\wedge} s: s \in S\right\} .
$$


Clearly $h t(T) \geq \omega \cdot \alpha+N$. We claim that $T \subseteq T_{\mu}^{I^{\prime}, \varepsilon / 2}$, thus $h t\left(T_{\mu}^{I, \varepsilon}\right) \geq \omega \cdot \alpha+N$ and we are done. Evidently, every element of a sequence $\left(n_{0}, \ldots, n_{N-1}\right)^{\wedge} s \in S$ is at least twice as big as its predecessor. Also, we have

$$
\mu\left(\left\{x: n_{0} x \notin I^{\prime} \& \cdots \& n_{N-1} x \notin I^{\prime}\right)\right\} \geq 1-\varepsilon / 2
$$

and, if $\left(n_{0}, \ldots, n_{N-1}, n_{N}, \ldots, n_{N+m}\right) \in T$, then

$$
\begin{aligned}
\mu\left(\left\{x: n_{N} x \notin I^{\prime} \& \cdots \& n_{N+m} x \notin I^{\prime}\right\}\right. \\
\\
\geq \mu\left(\left\{x: n_{N} x \notin I \& \cdots \& n_{N+m} x \notin I\right\}\right) \geq \varepsilon .
\end{aligned}
$$

Therefore

$$
\mu\left(\left\{x: n_{0} x \notin I^{\prime} \& \cdots \& n_{N+m} x \notin I^{\prime}\right\}\right) \geq \varepsilon / 2
$$

and our proof is complete.

As mentioned before, we may now define $h(\mu)$ for $\mu \in H^{\perp}$ by $h^{\prime}(\mu)=\omega \cdot h(\mu)$. The following fact establishes the basic definability properties of this rank.

PROPOSITIN 1.4. The rank $h: H^{\perp} \rightarrow \omega_{1}$ is a $\Pi_{1}^{1}$-rank on the $\Pi_{1}^{1}$ set $H^{\perp}$.

Proof. Recall that if $X$ is a Polish space, $P \subseteq X$ is a $\Pi_{1}^{1}$ set in $X$ and $\varphi: P \rightarrow \omega_{1}$ is a rank on $P$, then we say $\varphi$ is a $\Pi_{1}^{1}$-rank if, letting $\varphi(x)=\omega_{1}$ for all $x \notin P$, we have that the relations

$$
\begin{aligned}
& x \leq_{\varphi}^{*} y \Leftrightarrow x \in P \& \varphi(x) \leq \varphi(y), \\
& x<_{\varphi}^{*} y \Leftrightarrow x \in P \& \varphi(x)<\varphi(y) \quad(\Leftrightarrow \varphi(x)<\varphi(y))
\end{aligned}
$$

are $\Pi_{1}^{1}$ (as subsets of $X^{2}$ ).

A typical example of a $\Pi_{1}^{1}$-rank is the following (see [KL, V.1]): Identifying trees on Seq $\mathbf{N}^{*}$ with their characteristic functions, we can view them as members of the space $2^{\operatorname{Seq} \mathbf{N}^{*}}\left(=\{0,1\}^{\operatorname{Seq} \mathbf{N}^{*}}\right)$. Let $W F \subseteq 2^{\operatorname{Seq} \mathbf{N}^{*}}$ be the set of well-founded trees on Seq $\mathbf{N}^{*}$. Then $W F$ is $\Pi_{1}^{1}$. Moreover, the rank $T \mapsto h t(T)$ is a $\Pi_{1}^{1}$-rank on $W F$.

Going back to $h$ now, notice that for $\mu, \nu \in H^{\perp}, h(\mu) \leq h(\nu) \Leftrightarrow h^{\prime}(\mu) \leq h^{\prime}(\nu)$, so it is enough to show that $h^{\prime}$ is a $\Pi_{1}^{1}$-rank on $H^{\perp}$. Again let $h^{\prime}(\mu)=\omega_{1}$ if $\mu \notin H^{\perp}$. Then

$$
\begin{aligned}
\mu \leq_{h^{\prime}}^{*} \nu & \Leftrightarrow \mu \in H^{\perp} \& h^{\prime}(\mu) \leq h^{\prime}(\nu) \\
& \Leftrightarrow \mu \in H^{\perp} \& \forall I, \varepsilon \exists J, \delta\left[\operatorname{ht}\left(T_{\mu}^{I, \varepsilon}\right) \leq \operatorname{ht}\left(T_{\nu}^{J, \delta}\right)\right] \\
& \Leftrightarrow \mu \in H^{\perp} \& \forall I, \varepsilon \exists J, \delta\left[T_{\mu}^{I, \varepsilon} \in W F \& \operatorname{ht}\left(T_{\mu}^{I, \varepsilon}\right) \leq \operatorname{ht}\left(T_{\nu}^{J, \delta}\right)\right]
\end{aligned}
$$

and

$$
\begin{aligned}
\mu<{ }_{h^{\prime}}^{*} \nu & \Leftrightarrow \mu \in H^{\perp} \& h^{\prime}(\mu)<h^{\prime}(\nu) \\
& \Leftrightarrow \mu \in H^{\perp} \& \exists J, \delta \forall I, \varepsilon\left[T_{\mu}^{I, \varepsilon} \in W F \& \operatorname{ht}\left(T_{\mu}^{I, \varepsilon}\right)<\operatorname{ht}\left(T_{\nu}^{J, \delta}\right)\right] .
\end{aligned}
$$

Since $\Pi_{1}^{1}$ sets are closed under countable intersections and unions as well as Borel pre-images and ht is a $\Pi_{1}^{1}$-rank on $W F$, it is enough to show that for each fixed pair $I, \varepsilon$, the map $\mu \mapsto T_{\mu}^{I, \varepsilon}$ is a Borel map from $\operatorname{PROB}(\mathbf{T})$ into $2^{\text {Seq } \mathbf{N}^{*}}$. Since $2^{\text {Seq } \mathbf{N}^{*}}$ has the product topology, this reduces to showing that for each fixed $s=$ $\left(n_{0}, \ldots, n_{k}\right)$ with $2 n_{i} \leq n_{i+1}$, the set

$$
\left\{\mu \in \operatorname{PROB}(\mathbf{T}): s \in T_{\mu}^{I, \varepsilon}\right\}=\{\mu \in \operatorname{PROB}(\mathbf{T}): \mu(F) \geq \varepsilon\},
$$


where $F=\bigcap_{i=0}^{k}\left\{x: n_{i} x \notin I\right\}$, is a Borel set. Since $F$ is closed, let $F=\bigcap_{n} V_{n}, V_{n}$ decreasing and open in $\mathbf{T}$. Then

$$
\mu(F) \geq \varepsilon \Leftrightarrow \forall \varepsilon^{\prime}<\varepsilon \forall n\left(\mu\left(V_{n}\right)>\varepsilon^{\prime}\right),
$$

so actually this set is a $G_{\delta}$ and we are done.

2. Measures of low rank. Let $A=\left\{f \in C(\mathbf{T}): \hat{f} \in l^{1}(\mathbf{Z})\right\}$ with $\|f\|_{A}=$ $\|\hat{f}\|_{l^{1}}$. For $f \in A, \mu \in R$, and $\varepsilon>0$, define

$$
N(f, \varepsilon)=\min \left\{N \geq 0: \sum_{|n|>N}|\hat{f}(n)|<\varepsilon\right\}
$$

and

$$
N^{\prime}(\mu, \varepsilon)=\min \{N \geq 0:|n|>N \Rightarrow|\hat{\mu}(n)|<\varepsilon\} .
$$

We write $\left(T_{m} f\right)(x)=f(m x)$. Thus, $\left\|T_{m} f\right\|_{A}=\|f\|_{A}$ and $N\left(T_{m} f, \varepsilon\right)=m N(f, \varepsilon)$ for $m \geq 1$.

LEMMA 2.1. For $f, g \in A$ and $\varepsilon>0$,

$$
N(f g, \varepsilon) \leq N\left(f, \frac{\varepsilon}{2\|g\|_{A}}\right)+N\left(g, \frac{\varepsilon}{2\|f\|_{A}}\right) .
$$

PROOF. Denote the terms on the right by $N_{1}$ and $N_{2}$. If $|k+l|>N_{1}+N_{2}$, then $|k|>N_{1}$ or $|l|>N_{2}$. Therefore

$$
\begin{aligned}
\sum_{|n|>N_{1}+N_{2}}|\widehat{f g}(n)| & =\sum_{|n|>N_{1}+N_{2}}\left|\sum_{k+l=n} \hat{f}(k) \hat{g}(l)\right| \\
& \leq \sum_{\substack{|k|>N_{1} \\
l \in \mathbf{Z}}}|\hat{f}(k) \hat{g}(l)|+\sum_{\substack{|l|>N_{2} \\
k \in \mathbf{Z}}}|\hat{f}(k) \hat{g}(l)|<\varepsilon .
\end{aligned}
$$

LEMMA 2.2. For $f, g \in A, \mu \in R$, and

$$
n>2 \max \left(N\left(f, \frac{\varepsilon}{2\|g\|_{A}}\right), N^{\prime}\left(\mu, \frac{\varepsilon}{2\|f\|_{A}\|g\|_{A}}\right)\right),
$$

we have

$$
\left|\int f \cdot T_{n} g d \mu-\int f d \mu \cdot \hat{g}(0)\right|<\varepsilon .
$$

ProOF. Indeed, we have

$$
\begin{aligned}
& \left|\int f \cdot T_{n} g d \mu-\int f d \mu \cdot \hat{g}(0)\right|=\left|\sum_{r \neq 0} \hat{g}(-r) \sum_{l} \hat{f}(-l) \hat{\mu}(n r+l)\right| \\
& \quad \leq \sum_{r \neq 0}|\hat{g}(-r)|\left\{\sum_{|l| \leq n / 2}|\hat{f}(-l) \hat{\mu}(n r+l)|+\sum_{|l|>n / 2}|\hat{f}(-l) \hat{\mu}(n r+l)|\right\} \\
& \quad<\varepsilon
\end{aligned}
$$

since if $|l| \leq n / 2$ and $r \neq 0,|n r+l| \geq n / 2$. 
Lemma 2.3. If $\mu \in R, f \in A,|\hat{f}(0)| \leq 1,\|f\|_{A} \geq 1, K \geq 2, m_{1}, m_{2}, \ldots, m_{K} \in$ $\mathbf{N}^{*}$,

$$
\frac{m_{j+1}}{m_{j}} \geq 2 \max \left(K N\left(f, \frac{\varepsilon}{2^{K-1} K\|f\|_{A}^{K-1}}\right), N^{\prime}\left(\mu, \frac{\varepsilon}{2 K\|f\|_{A}^{K}}\right)\right)
$$

for $1 \leq j<K$, then

$$
\left|\int \prod_{j=1}^{K} T_{m_{j}} f d \mu-\hat{f}(0)^{K}\right|<\varepsilon .
$$

ProOF. Let $F_{k}=\prod_{j=1}^{k} T_{m_{j}} f$. We shall show that

$$
\left|\int F_{k+1} d \mu-\int F_{k} d \mu \cdot \hat{f}(0)\right|<\frac{\varepsilon}{K}
$$

for $0 \leq k<K$, where $F_{0} \equiv 1$. Multiplying both sides by $|\hat{f}(0)|^{K-k-1}$ and adding over $k$ yields the desired inequality. Now (2.1) will follow if

$$
m_{k+1} \geq 2 \max \left(N\left(F_{k}, \frac{\varepsilon}{2 K\|f\|_{A}}\right), N^{\prime}\left(\mu, \frac{\varepsilon}{2 K\|f\|_{A}^{k+1}}\right)\right) .
$$

That $m_{k+1}$ is at least twice the second term follows immediately from the hypothesis. Thus, it suffices to show that

$$
m_{k} K N\left(f, \frac{\varepsilon}{2^{K-1} K\|f\|_{A}^{K-1}}\right) \geq N\left(F_{k}, \frac{\varepsilon}{2 K\|f\|_{A}}\right) .
$$

By repeated application of Lemma 2.1, we obtain

$$
\begin{aligned}
N\left(F_{k},\right. & \left.\frac{\varepsilon}{2 K\|f\|_{A}}\right) \leq N\left(F_{k-1}, \frac{\varepsilon}{4 K\|f\|_{A}^{2}}\right)+N\left(T_{m_{k}} f, \frac{\varepsilon}{4 K\|f\|_{A} \cdot\left\|F_{k-1}\right\|_{A}}\right) \\
\leq & \cdots \leq N\left(T_{m_{1}} f, \frac{\varepsilon}{2^{k} K\|f\|_{A}^{k}}\right)+N\left(T_{m_{2}} f, \frac{\varepsilon}{2^{k} K\|f\|_{A}^{k}}\right) \\
& +N\left(T_{m_{3}} f, \frac{\varepsilon}{2^{k-1} K\|f\|_{A}^{k}}\right)+\cdots+N\left(T_{m_{k}} f, \frac{\varepsilon}{4 K\|f\|_{A}^{k}}\right) \\
\leq & k m_{k} N\left(f, \frac{\varepsilon}{2^{k} K\|f\|_{A}^{k}}\right) .
\end{aligned}
$$

This implies the desired inequality.

THEOREM 2.4. If $\mu \in R$, then $h(\mu)=1$; i.e., $R \subseteq H_{1}^{\perp}$.

PROOF. We shall show that $h^{\prime}(\mu) \leq \omega$. Let $I$ be a rational interval and $\varepsilon \in \mathbf{Q}^{+}$. Choose $f \in A$ so that $0 \leq f \leq 1, f=1$ on $I^{c}$, and $\hat{f}(0)<1$. Then $\|f\|_{A} \geq 1$ and $\mathbf{1}_{I^{c}} \leq f$. Choose $K$ so that $\hat{f}(0)^{K}<\varepsilon / 2$ and choose

$$
L \geq 2 K \log _{2} \max \left(2 K N\left(f, \frac{\varepsilon}{2^{K} K\|f\|_{A}^{K-1}}\right), 2 N^{\prime}\left(\mu, \frac{\varepsilon}{4 K\|f\|_{A}^{K}}\right)\right) .
$$

We claim that $h t\left(T_{\mu}^{I, \varepsilon}\right)<L$. Indeed, if $\left(m_{0}^{\prime}, \ldots, m_{L-1}^{\prime}\right) \in T_{\mu}^{I, \varepsilon}$, then there is a subsequence $\left\{m_{j}\right\}_{j=1}^{K}$ of $\left\{m_{i}^{\prime}\right\}_{i=0}^{L-1}$ such that

$$
\frac{m_{j+1}}{m_{j}} \geq 2 \max \left(K N\left(f, \frac{\varepsilon}{2^{K} K\|f\|_{A}^{K-1}}\right), N^{\prime}\left(\mu, \frac{\varepsilon}{4 K\|f\|_{A}^{K}}\right)\right) .
$$


Thus

$$
\begin{aligned}
\mu\{x: \forall i & \left.<L\left(m_{i}^{\prime} x \notin I\right)\right\} \leq \int \prod_{j=1}^{K} \mathbf{1}_{I^{c}}\left(m_{j} x\right) d \mu(x) \\
& \leq \int \prod_{1}^{K} f\left(m_{j} x\right) d \mu(x)<\hat{f}(0)^{K}+\frac{\varepsilon}{2}<\varepsilon
\end{aligned}
$$

a contradiction.

Let us say that a probability measure $\mu$ on $\mathbf{T}$ is quasisymmetric, or $\mu \in Q S$, if for some $C$, whenever $I_{1}$ and $I_{2}$ are adjacent intervals on $\mathbf{T}$ of the same length,

$$
\mu I_{1} \leq C \cdot \mu I_{2}
$$

(These measures are related to quasiconformal mappings; see [BA].) Note that every $\mu \in Q S$ is continuous. Examples include Riesz products (for background, see [K, p. 107])

$$
\mu=\prod_{k \geq 1}\left(1+\operatorname{Re}\left\{\alpha_{k} e\left(n_{k} x\right)\right\}\right)
$$

with $\varlimsup\left|\alpha_{k}\right|<1, n_{k+1} / n_{k} \geq 3$, and either $n_{k} \mid n_{k+1}$ or $n_{k+1} / n_{k} \rightarrow \infty$; we omit the proof. We do not know whether $Q S \subseteq H_{1}^{\perp}$, but we now show that $Q S \subseteq H_{\omega}^{\perp}$.

THEOREM 2.5. $Q S \subseteq H_{\omega}^{\perp}$.

Proof. Let $\mu \in Q S, I \subseteq \mathbf{T}$ be a rational interval, and $\varepsilon \in \mathbf{Q}^{+}$. Denote $T_{n}^{-1} I=\{x: n x \in I\}$. Then $T_{n}^{-1} I$ consists of $n$ equally spaced intervals separated by the $n$ intervals of $T_{n}^{-1} I^{c}$; the ratio of the lengths of adjacent intervals is $|I| /\left|I^{c}\right|$ and therefore the ratio of their $\mu$-measures is bounded by a constant, $C^{\prime}$, independent of $n$. It follows that if $A$ is a finite union of intervals, then

$$
\mu\left(A \cap T_{n}^{-1} I^{c}\right) \leq 2 C^{\prime} \mu\left(A \cap T_{n}^{-1} I\right)
$$

for all large $n$. Let $K$ be such that $\left(2 C^{\prime} /\left(1+2 C^{\prime}\right)\right)^{K}<\varepsilon$. We claim that ht $\left(T_{\mu}^{I, \varepsilon}\right)<$ $\omega \cdot K$, which will complete the proof.

Indeed, if $\operatorname{ht}\left(T_{\mu}^{I, \varepsilon}\right) \geq \omega \cdot K$, then there exist arbitrarily large $m$ such that $\operatorname{ht}\left((m), T_{\mu}^{I, \varepsilon}\right)>\omega \cdot(K-1)$. (Note that $s^{\wedge} t^{\wedge} u^{\wedge} v \in T_{\mu}^{I, \varepsilon} \Rightarrow t^{\wedge} v \in T_{\mu}^{I, \varepsilon}$.) Choose $m_{0}$ such that

$$
\operatorname{ht}\left(\left(m_{0}\right), T_{\mu}^{I, \varepsilon}\right)>\omega \cdot(K-1)
$$

and

$$
\mu\left(T_{m_{0}}^{-1} I^{c}\right) \leq 2 C^{\prime} \mu\left(T_{m_{0}}^{-1} I\right) .
$$

In a similar fashion, we may find inductively $m_{k}(1 \leq k \leq K-1)$ such that

$$
\operatorname{ht}\left(\left(m_{0}, m_{1}, \ldots, m_{k}\right), T_{\mu}^{I, \varepsilon}\right)>\omega \cdot(K-1-k)
$$

and

$$
\mu\left(E_{k-1} \cap T_{m_{k}}^{-1} I^{c}\right) \leq 2 C^{\prime} \mu\left(E_{k-1} \cap T_{m_{k}}^{-1} I\right)
$$

where

$$
E_{k}=\bigcap_{i=0}^{k} T_{m_{i}}^{-1} I^{c}
$$


Now (2.2) implies that

$$
\mu E_{k} \leq 2 C^{\prime}\left(\mu E_{k-1}-\mu E_{k}\right)
$$

or

$$
\mu E_{k} \leq \frac{2 C^{\prime}}{1+2 C^{\prime}} \mu E_{k-1} .
$$

Therefore

$$
\mu E_{K-1} \leq\left(\frac{2 C^{\prime}}{1+2 C^{\prime}}\right)^{K}<\varepsilon
$$

which contradicts the fact that $\left(m_{0}, m_{1}, \ldots, m_{K-1}\right) \in T_{\mu}^{I, \varepsilon}$.

3. Measures of large rank. For each $\alpha<\omega_{1}$, we shall construct a canonical tree $T_{\alpha}$ of height $\alpha$. Let $\mathscr{F}$ be the set of strictly increasing maps $f: \mathbf{N}^{*} \rightarrow \mathbf{N}^{*}$. Any $f \in \mathscr{F}$ induces a map $f^{*}$ on trees in the obvious way. Given $\left.\alpha<\omega_{1}, p \in\right] 0,1[$ and $f \in \mathscr{F}$, we shall construct a measure $\nu_{\alpha, p, f} \in H^{\perp}$ with $h^{\prime}$-rank at least $\alpha$. This shows that $H^{\perp}$ is not Borel.

Given a tree $T$, let

$$
(T)=\{n: \exists s(n \in s \in T)\},
$$

where, if $s=\left(n_{0}, n_{1}, \ldots, n_{k}\right)$, we write $n \in s \Leftrightarrow \exists i \leq k\left(n=n_{i}\right)$, and let $(T)^{c}=$ $\mathbf{N}^{*} \backslash(T)$. Define $f_{n}(x)=x^{2^{n+1}} \cdot 2^{2^{n}}$; note that if $x$ and $y$ are integral powers of 2 and $n \neq m$, then $f_{n}(x) \neq f_{m}(y)$. For each limit ordinal $\alpha<\omega_{1}$, choose a bijection $g_{\alpha}: \alpha \rightarrow \mathbf{N}$.

We construct our canonical trees $T_{\alpha}$ by induction, beginnning with $T_{0}=\{\varnothing\}$. It will turn out that $\left(T_{\alpha}\right)$ consists entirely of powers of 2 . If $T_{\gamma}$ have been constructed for $\gamma<\alpha$, let

$$
T_{\alpha}= \begin{cases}\left\{\left(2^{2}\right)^{\wedge} s: s \in f_{0}^{*} T_{\beta}\right\} \cup\{\varnothing\} & \text { if } \alpha=\beta+1, \\ \bigcup_{\beta<\alpha} f_{g_{\alpha}(\beta)}^{*} T_{\beta} & \text { if } \alpha \text { is a limit. }\end{cases}
$$

It is clear that $\operatorname{ht}\left(T_{\alpha}\right)=\alpha$.

Given a set $A \subseteq \mathrm{N}^{*}$, define the Bernoulli convolution

$$
\lambda_{A}=\underset{n \in A}{*}\left[\frac{1}{2} \delta(0)+\frac{1}{2} \delta\left(2^{-n}\right)\right],
$$

where $\delta(x)$ represents the Dirac measure concentrated at $x$. For $\left.\alpha<\omega_{1}, p \in\right] 0,1[$, $f \in \mathscr{F}$, we construct preliminary measures $\mu_{\alpha, p, f}$ by induction. Let $\mu_{0, p, f}=\delta(0)$ for all $p, f$. If $\mu_{\gamma, p, f}$ have been constructed for all $\left.\gamma<\alpha, p \in\right] 0,1[$, and $f \in \mathscr{F}$, define

where

$$
\mu_{\alpha, p, f}=\sqrt{p} \mu_{\alpha, p, f}^{(0)}+(1-\sqrt{p}) \lambda_{\left(f^{*} T_{\alpha}\right)},
$$

$$
\mu_{\alpha, p, f}^{(0)}= \begin{cases}\mu\left\langle\beta, \sqrt{p}, f \circ f_{0}\right\rangle & \text { if } \alpha=\beta+1, \\ \beta_{<\alpha}^{*} \mu\left\langle\beta, \sqrt{p}, f \circ f_{g_{\alpha}(\beta)}\right\rangle & \text { if } \alpha \text { is a limit; }\end{cases}
$$

here, and below, we are using the notation $\langle\cdots\rangle$ for subscripts.

Finally, define

$$
\nu_{\alpha, p, f}=\lambda_{\left(f^{*} T_{\alpha}\right)^{c}} * \mu_{\alpha, p, f} .
$$

The reader should have in mind the following probabilistic interpretation of $\nu_{\alpha, p, f}$. First define i.i.d. random variables $Y_{n}$ for $n \geq 1$ which take the values 
0,1 each with probability $1 / 2$. For $A \subseteq \mathrm{N}^{*}$, let $Y_{A}=\sum_{n \in A} Y_{n} 2^{-n}$. Let $Z_{p}$ be independent random variables for $0<p<1$, independent also of $\left\{Y_{n}\right\}$, which take the values 0,1 with probabilities $\sqrt{p}$ and $1-\sqrt{p}$, respectively. We define random variables $X_{\alpha, p, f}$ by induction. Let $X_{0, p, f} \equiv 0$. To define $X_{\alpha . p, f}$ given $X_{\gamma, q, g}$ for $\gamma<\alpha$, first let $\left\{\tilde{X}_{\gamma, q, g}: \gamma<\alpha, q \in\right] 0,1[, g \in \mathscr{F}\}$ be independent copies of $\left\{X_{\gamma, q, g}\right\}$ which are also independent of $\left\{Z_{p}\right\}$ and $\left\{Y_{n}\right\}$. Now define

$$
X_{\alpha, p, f}=\left(1-Z_{p}\right) X_{\alpha, p, f}^{(0)}+Z_{p} Y_{\left(f * T_{\alpha}\right)},
$$

where

Finally, define

$$
X_{\alpha, p, f}^{(0)}= \begin{cases}\tilde{X}_{\beta, \sqrt{p}, f^{\circ} f_{0}} & \text { if } \alpha=\beta+1, \\ \sum_{\beta<\alpha} \tilde{X}\left\langle\beta, \sqrt{p}, f \circ f_{g_{\alpha}(\beta)}\right\rangle & \text { if } \alpha \text { is a limit. }\end{cases}
$$

$$
W_{\alpha, p, f}=X_{\alpha, p, f}+Y_{\left(f^{*} T_{\alpha}\right)^{c}} .
$$

Then the distributions of $Y_{A}, X_{\alpha, p, f}, X_{\alpha, p, f}^{(0)}$, and $W_{\alpha, p, f}$ are $\lambda_{A}, \mu_{\alpha, p, f}, \mu_{\alpha, p, f}^{(0)}$ and $\nu_{\alpha, p, f}$, respectively. One may show by induction that $X_{\alpha, p, f}$ can be written in the form

$$
X_{\alpha, p, f}=\sum_{n \geq 1} U_{n} 2^{-n}
$$

for some $\{0,1\}$-valued random variables $U_{n}$ with $U_{n} \equiv 0$ if $n \notin\left(f^{*} T_{\alpha}\right)$.

The following proposition shows that $f^{*} T_{\alpha} \subseteq T_{\nu_{\alpha, p, f}}^{1 / 2,1[, p}$. Combined with Theorem 3.2, this shows that $h^{\prime}\left(\nu_{\alpha, p, f}\right) \geq \alpha$.

Proposition 3.1. $\left.\forall \alpha<\omega_{1} \forall p \in\right] 0,1\left[\forall f \in \mathscr{F} \forall s \in f^{*} T_{\alpha}\right.$

$$
\nu_{\alpha, p, f}\left(E_{s}\right)=\mu_{\alpha, p, f}\left(E_{s}\right) \geq p
$$

and

$$
\mu_{\alpha, p, f}\left(E_{\left(f^{*} T_{\alpha}\right)^{c}}\right)=1
$$

where for $A \subseteq \mathrm{N}^{*}$,

$$
E_{A}=\left\{x \in \mathbf{T}: \forall n \in A\left(2^{n-1} x \notin\left[\frac{1}{2}, 1[)\right\} .\right.\right.
$$

Proof. We proceed by induction. Condition (3.2) is easily established, so we concentrate on (3.1). It is clear that $\nu_{\alpha, p, f} E_{s}=\mu_{\alpha, p, f} E_{s}$. Now (3.1) is trivial for $\alpha=0$, so suppose it holds for all $\gamma<\alpha$. If $\alpha=\beta+1$ and $s \in f^{*} T_{\alpha}$, then $s=\left(f\left(2^{2}\right)\right)^{\wedge} s_{0}$ for some $s_{0} \in\left(f \circ f_{0}\right)^{*} T_{\beta}$. Therefore

$$
\begin{aligned}
\mu_{\alpha, p, f}\left(E_{s}\right) & \geq \sqrt{p} \mu\left\langle\beta, \sqrt{p}, f \circ f_{0}\right\rangle\left(E_{s}\right) \\
& \geq \sqrt{p} \mu\left\langle\beta, \sqrt{p}, f \circ f_{0}\right\rangle\left(E_{s_{0}} \cap E\left\langle\left(\left(f \circ f_{0}\right)^{*} T_{\beta}\right)^{c}\right\rangle\right) \\
& =\sqrt{p} \mu\left\langle\beta, \sqrt{p}, f \circ f_{0}\right\rangle\left(E_{s_{0}}\right) \quad(\text { by }(3.2)) \\
& >\sqrt{p} \cdot \sqrt{p}=p
\end{aligned}
$$

by the inductive hypothesis. On the other hand, if $\alpha$ is a limit and $s \in f^{*} T_{\alpha}$, then $s \in\left(f \circ f_{g_{\alpha}(\beta)}\right)^{*} T_{\beta}$ for some $\beta<\alpha$. Therefore

$$
\mu_{\alpha, p, f} E_{s} \geq \sqrt{p} \mu_{\alpha, p, f}^{(0)} E_{s}=\sqrt{p} \mu\left\langle\beta, \sqrt{p}, f \circ f_{g_{\alpha}(\beta)}\right\rangle\left(E_{s}\right) \geq p .
$$

It remains to prove that $\nu_{\alpha, p, f} \in H^{\perp}$, for which we use the methods of [L3]. 
THEOREM 3.2. $\left.\forall \alpha<\omega_{1} \forall p \in\right] 0,1\left[\forall f \in \mathscr{F} \nu_{\alpha, p, f} \in H^{\perp}\right.$.

Proof. For a sequence $\left\{m_{j}\right\} \subseteq \mathbf{Z}$, a measure $\mu \in M(\mathbf{T})$, and measures $\sigma_{x, \mu} \in$ $M(\mathbf{T})(x \in \mathbf{T})$, we shall write $\left\{m_{j}\right\} \sim \sigma_{x, \mu}$ if for all $r \in \mathbf{Z}$,

$$
e\left(-r m_{j} x\right) \rightarrow \hat{\sigma}_{x, \mu}(r) \quad \text { weak }^{*} \text { in } L^{\infty}(\mu) .
$$

According to Theorem 13 of [L3], if $m_{j} \rightarrow \infty$ and $\left\{m_{j}\right\} \sim \sigma_{x, \mu}$, then $\operatorname{supp} \sigma_{x, \mu}=$ T $\mu$-a.e. iff $\mu$ annihilates all $H$-sets based on any subsequence of $\left\{m_{j}\right\}$.

By the method of proof of Theorem 16 of [L3], we see that to show that $\nu_{\alpha, p, f} \in$ $H^{\perp}$, it is enough to show that

$$
\operatorname{supp} \sigma\left\langle x, \nu_{\alpha, p, f}\right\rangle=\mathbf{T} \quad \nu_{\alpha, p, f} \text {-a.e. }
$$

when $\left\{2^{n_{j}-1} x\right\} \sim \sigma\left\langle x, \nu_{\alpha, p, f}\right\rangle$ for some increasing sequence $\left\{n_{j}\right\} \subseteq\left(f^{*} T_{\alpha}\right)$; in particular, we can take $\alpha \geq \omega$. Furthermore, by taking a subsequence, if necessary, $\left\{e\left(-r 2^{n_{j}-1} x\right)\right\}$ has weak ${ }^{*}$ limits in $L^{\infty}\left(\lambda_{\left[f^{*} T_{\alpha}\right]^{c}}\right)$ and $L^{\infty}\left(\mu_{\alpha, p, f}\right)$ as well, and

$$
\sigma\left\langle x+y, \nu_{\alpha, p, f}\right\rangle=\sigma\left\langle x, \lambda_{\left(f^{*} T_{\alpha}\right)^{c}}\right\rangle * \sigma\left\langle y, \mu_{\alpha, p, f}\right\rangle \quad \lambda_{\left(f^{*} T_{\alpha}\right)^{c}} \otimes \mu_{\alpha, p, f^{-a . e . ~}[(x, y)]}
$$

(see [L4]). It is easy to calculate that

$$
\sigma\left\langle x, \lambda_{\left(f^{*} T_{\alpha}\right)^{c}}\right\rangle=\lambda_{\{n: n \geq 2\}} \text { a.e. }
$$

Hence it suffices to show that $\left.\exists q_{y} \in\right] 0,1[$ such that

$$
\sigma\left\langle y, \mu_{\alpha, p, f}\right\rangle=\left(1-q_{y}\right) \delta(0)+q_{y} \delta\left(2^{-1}\right) \quad \text { a.e. }
$$

Now

$$
\operatorname{supp} \mu_{\alpha, p, f}=\left\{\sum_{n \in\left(f^{*} T_{\alpha}\right)} a_{n} 2^{-n}: a_{n}=0,1\right\},
$$

whence (3.3) holds for some $q_{y} \in[0,1]$. Now $q_{y}=\frac{1}{2} \lambda_{\left(f^{*} T_{\alpha}\right)^{-a . e .}}$, so it remains to consider $\mu_{\alpha, p, f}^{(0)}$.

We proceed by induction, beginning with the case of a limit ordinal $\alpha$, which includes the possibility that $\alpha=\omega$. Suppose first that $\left\{n_{j}\right\}$ intersects infinitely many $\left(\left(f \circ f_{g_{\alpha}(\beta)}\right)^{*} T_{\beta}\right)$. Then we may assume that $n_{j} \in\left(\left(f \circ f_{g_{\alpha}\left(\beta_{j}\right)}\right)^{*} T_{\beta_{j}}\right)$ with $\left\{\beta_{j}\right\}$ distinct. Now if $q_{y} \in\{0,1\}$ on a set of positive $\mu_{\alpha, p, f}^{(0)}$-measure, then by Theorem 13 of [L3] quoted above, there is an $H$-set based on a subsequence of $\left\{2^{n_{j}-1}\right\}$ of positive $\mu_{\alpha, p, f}^{(0)}$-measure. By relabelling, we take this subsequence to be the whole sequence. Furthermore, because of (3.4), we conclude that $\mu_{\alpha, p, f}^{(0)} E^{(a)}>0$ for either $a=0,1$, where $E^{(a)}=\bigcap_{j<\infty} E_{j}^{(a)}$,

$$
E_{j}^{(a)}=\left\{x: 2^{n_{j}-1} x \in \frac{a}{2}+\left[0, \frac{1}{2}[\} .\right.\right.
$$

But $E_{j}^{(a)}$ are $\mu_{\alpha, p, f}^{(0)}$-independent since $\left\{\beta_{j}\right\}$ are distinct. Also,

$$
\mu_{\alpha, p, f}^{(0)} E_{j}^{(0)}=\mu\left\langle\beta_{j}, \sqrt{p}, f \circ f_{g_{\alpha}\left(\beta_{j}\right)}\right\rangle\left(E_{j}^{(0)}\right) \geq \sqrt{p}
$$

and

$$
\begin{aligned}
\mu_{\alpha, p, f}^{(0)} E_{j}^{(1)} & =\mu\left\langle\beta_{j}, \sqrt{p}, f \circ f_{g_{\alpha}\left(\beta_{j}\right)}\right\rangle\left(E_{j}^{(1)}\right) \\
& \geq\left(1-p^{1 / 4}\right) \lambda\left\langle\left(\left(f \circ f_{g_{\alpha}\left(\beta_{j}\right)}\right)^{*} T_{\beta_{j}}\right)\right\rangle\left(E_{j}^{(1)}\right)=\left(1-p^{1 / 4}\right) \cdot \frac{1}{2}
\end{aligned}
$$


Since $E_{j}^{(0)}$ and $E_{j}^{(1)}$ are complementary events, it follows that $\mu_{\alpha, p, f}^{(0)} E^{(a)}=0$, a contradiction.

Now in the second case, $\left\{n_{j}\right\}$ intersects only finitely many $\left(\left(f \circ f_{g_{\alpha}(\beta)}\right)^{*} T_{\beta}\right)$. Here we may then assume that all $n_{j} \in\left(\left(f \circ f_{g_{\alpha}\left(\beta_{0}\right)}\right)^{*} T_{\beta_{0}}\right)$ for some $\beta_{0}$. We have

$$
\sigma\left\langle x+y, \mu_{\alpha, p, f}^{(0)}\right\rangle=\sigma\left\langle x, \mu\left\langle\beta_{0}, \sqrt{p}, f \circ f_{g_{\alpha}\left(\beta_{0}\right)}\right\rangle\right\rangle * \sigma\left\langle y \underset{\substack{\beta<\alpha \\ \beta \neq \beta_{0}}}{*} \mu\left\langle\beta, \sqrt{p}, f \circ f_{g_{\alpha}(\beta)}\right\rangle\right\rangle
$$

a.e. $[(x, y)]$ and by the inductive hypothesis, the first term on the right equals $q_{x}^{\prime} \delta(0)+\left(1-q_{x}^{\prime}\right) \delta\left(\frac{1}{2}\right)$ with $0<q_{x}^{\prime}<1$ a.e. $[x]$. Hence (3.3) holds with $0<q_{x}<1$.

Finally, in case $\alpha=\beta+1$ is a successor ordinal, we have

$$
\sigma\left\langle x, \mu_{\alpha, p, f}\right\rangle=\sigma\left\langle x, \mu\left\langle\beta, \sqrt{p}, f \circ f_{0}\right\rangle\right\rangle \quad \mu_{\alpha, p, f}^{(0)} \text {-a.e. }
$$

whence the desired result follows directly from the inductive hypothesis.

4. Measures annihilating Dirichlet sets. Every Dirichlet set is evidently an $H$-set: $D \subseteq H$. Thus $D^{\perp} \supseteq H^{\perp}$. It turns out that $D^{\perp}$ is again $\Pi_{1}^{1}$ non-Borel. The method of proof is similar to that for $H^{\perp}$, and we shall only outline it here.

First one checks that $D$ is Borel, in fact $\Pi_{2}^{0}$ (i.e., $G_{\delta}$ ). Thus, $D^{\perp}$ is $\Pi_{1}^{1}$. Given $\mu \in \operatorname{PROB}(\mathbf{T})$ and $\varepsilon \in \mathbf{Q}^{+}$, we define a tree $T_{\mu}^{\varepsilon}$ on $\operatorname{Seq}\left(\mathbf{N}^{*} \times \mathbf{Q}^{+}\right)$as follows:

$$
\begin{aligned}
T_{\mu}^{\varepsilon}=\left\{\left(\left(n_{0}, r_{0}\right),\left(n_{1}, r_{1}\right), \ldots,\left(n_{k}, r_{k}\right)\right): \forall i<k\left(n_{i+1} \geq 2 n_{i} \& r_{i+1}<r_{i}\right)\right. \\
\\
\left.\& \mu\left\{x \in \mathbf{T}: \forall i \leq k\left(\left\|n_{i} x\right\| \leq r_{i}\right)\right\} \geq \varepsilon\right\} .
\end{aligned}
$$

Thus, $\mu \in D^{\perp} \Leftrightarrow \forall \varepsilon$ ( $T_{\mu}^{\varepsilon}$ is well founded). If we define

$$
h_{D}^{\prime}(\mu)=\sup \left\{h t\left(T_{\mu}^{\varepsilon}\right)+1: \varepsilon \in \mathbf{Q}^{+}\right\},
$$

then $h_{D}^{\prime}(\mu)$ is a limit ordinal, $\omega \cdot h_{D}(\mu)$. One proves as before that $h_{D}: D^{\perp} \rightarrow \omega_{1}$ is a $\Pi_{1}^{1}$-rank on $D^{\perp}$. Note that $h \geq h_{D}$, so that all measures of $h$-rank one are also of $h_{D}$-rank one.

We now construct measures of arbitrarily large $h_{D}$-rank. Given $A \subseteq \mathbf{N}^{*}$, denote

$$
A_{2}=\{k \in \mathrm{N}: \exists n \in A n \leq k<2 n\} .
$$

We define $\tau_{\alpha, p, f}$ by induction, using the notation of $\S 3$ : let $\tau_{0, p, f}=\delta(0)$ and

$$
\tau_{\alpha, p, f}=\sqrt{p} \tau_{\alpha, p, f}^{(0)}+(1-\sqrt{p}) \lambda_{\left(f^{*} T_{\alpha}\right)_{2}}
$$

where

$$
\tau_{\alpha, p, f}^{(0)}= \begin{cases}\tau\left\langle\beta, \sqrt{p}, f \circ f_{0}\right\rangle & \text { if } \alpha=\beta+1 \\ \beta_{\beta<\alpha}^{*} \tau\left\langle\beta, \sqrt{p}, f \circ f_{g_{\alpha}(\beta)}\right\rangle & \text { if } \alpha \text { is a limit. }\end{cases}
$$

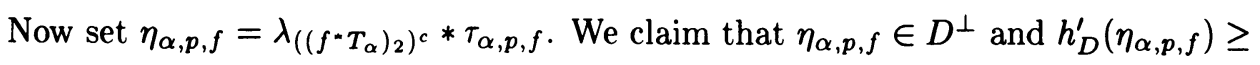
$\alpha$.

One first shows that if $s \in f^{*} T_{\alpha}$, then

$$
\eta_{\alpha, p, f}\left\{x \in \mathbf{T}: \forall n \in s 2^{n-1} x \in\left[0,2^{-n}[\} \geq p .\right.\right.
$$

Next, notice that $\mu \in D^{\perp}$ iff $\sigma_{x, \mu} \neq \delta(0) \mu$-a.e. whenever $\left\{m_{j}\right\} \sim \sigma_{x, \mu}$ (see [L4]). As before,

$$
\sigma\left\langle x+y, \eta_{\alpha, p, f}\right\rangle=\sigma\left\langle x, \lambda_{\left(f^{*} T_{\alpha}\right)_{2}^{c}}\right\rangle * \sigma\left\langle y, \tau_{\alpha, p, f}\right\rangle \quad \text { a.e. }[(x, y)]
$$


Suppose that the left-hand side is $\delta(0)$ on a set of positive measure. Then both members of the right-hand side are Dirac measures on sets of positive measure (since both are probability measures). Also, $\lambda_{\left(f^{*} T_{\alpha}\right)_{2}^{c}}$ is monotrochic (see the proof of Proposition 6.4.5, p. 173, of [GM]), whence if $\sigma\left\langle x, \lambda_{\left(f^{*} T_{\alpha}\right)_{2}^{c}}\right\rangle$ is a Dirac measure on a set of positive $\lambda_{\left(f^{*} T_{\alpha}\right)_{2}^{c}}$-measure and we denote $\chi(x)=\left(\sigma\left\langle x, \lambda_{\left(f^{*} T_{\alpha}\right)_{2}^{c}}\right\rangle\right)^{\wedge}(1)$,

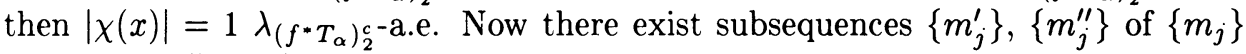
such that $m_{j}^{\prime \prime}>m_{j}^{\prime}$ and

$$
e\left(-\left(m_{j}^{\prime \prime}-m_{j}^{\prime}\right) x\right) \rightarrow\left|\hat{\sigma}_{x, \mu}(1)\right|^{2} \quad \text { weak }^{*} \text { in } L^{\infty}(\mu)
$$

for $\mu=\eta_{\alpha, p, f}, \lambda_{\left(f^{*} T_{\alpha}\right)_{2}^{c}}$, and $\tau_{\alpha, p, f}$ (see Lemme I.5, p. 17, of [HMP]). Thus, if $\left\{m_{j}^{\prime \prime}-m_{j}^{\prime}\right\} \sim \sigma_{x, \mu}^{\prime}$ and $\sigma_{x, \mu}$ is a Dirac measure on a set $E$ of positive $\mu$-measure, then $\sigma_{x, \mu}^{\prime}=\delta(0)$ on almost all of $E$, where $\mu$ is any of the above three measures. Hence we may relabel $m_{j}^{\prime \prime}-m_{j}^{\prime}$ as $m_{j}$ without loss of generality. But now we have $\chi \geq 0$, whence $\chi(x)=1$ a.e., so that $\hat{\lambda}_{\left(f^{*} T_{\alpha}\right)_{2}^{c}}\left(m_{j}\right) \rightarrow 1$, which is the same as $m_{j}=a_{j} 2^{n_{j}}$ for some $n_{j} \in\left(f^{*} T_{\alpha}\right)$ and $a_{j} \in \mathbf{Z}$ with $a_{j} 2^{-n_{j}} \rightarrow 0$. An inductive argument shows that for such $\left\{m_{j}\right\}, \sigma\left\langle y, \tau_{\alpha, p, f}\right\rangle \neq \delta(0)$ a.e., which contradicts our supposition and completes the proof that $\eta_{\alpha, p, f} \in D^{\perp}$.

\section{REFERENCES}

[BA] A. Beurling and L. Ahlfors, The boundary correspondence under quasiconformal mappings, Acta Math. 96 (1956), 125-142.

[GM] C. C. Graham and O. C. McGehee, Essays in commutative harmonic analysis, SpringerVerlag, New York, 1979.

[HMP] B. Host, J.-F. Méla and F. Parreau, Analyse harmonique des mesures, Astérisque 135-136 (1986).

[K] Y. Katznelson, An introduction to harmonic analysis, 2nd corrected ed., Dover, New York, 1976.

[KL] A. S. Kechris and A. Louveau, Descriptive set theory and the structure of sets of uniqueness, London Math. Soc. Lecture Notes 128, Cambridge Univ. Press, Cambridge, 1987.

[LP] L.-A. Lindahl and F. Poulsen (eds.), Thin sets in harmonic analysis, Dekker, New York, 1971.

[L1] R. Lyons, A characterization of measures whose Fourier-Stieltjes transforms vanish at infinity, Ph.D. Thesis, Univ. of Michigan, 1983.

[L2] _ , The size of some classes of thin sets, Studia Math. 86 (1987), 59-78.

[L3] _ Mixing and asymptotic distribution modulo 1, Ergodic Theory Dynamical Systems (to appear).

[L4] _ The local structure of some measure-algebra homomorphisms, preprint.

[L5] __ Singular measures with spectral gaps, Proc. Amer. Math. Soc. 104 (1988), 86-88.

[R1] A. Rajchman, Sur l'unicité du développment trigonométrique, Fund. Math. (Varsovie) 3 (1922), 287-302. Rectification et addition à ma note "Sur l'unicité... ", Fund. Math. 4 (1923), 366-367.

[R2] __ Sur la multiplication des séries trigonométriques et sur une classe remarquable d'ensembles fermés, Math. Ann. 95 (1925), 389-408.

[Z] A. Zygmund, Trigonometric series, 2nd ed. reprinted, Volumes I, II, Cambridge Univ. Press, Cambridge, 1979.

Department of Mathematics, California institute of Technology, PasaDENA, CALIFORNIA 91125

Department of Mathematics, Stanford University, Stanford, California 94305 